\title{
Exchange interaction of electrons with Mn in hybrid AISb/InAs/ZnMnTe structures
}

\author{
Ya. V. Terent'ev ${ }^{1,2}$ C. Zoth, ${ }^{1}$ V. V. Bel'kov, ${ }^{1,2}$ P. Olbrich, ${ }^{1}$ C. Drexler,${ }^{1}$ V. Lechner, ${ }^{1}$ P. Lutz, ${ }^{1}$ \\ M. S. Mukhin ${ }^{2}$ S. A. Tarasenko, ${ }^{2}$ A. N. Semenov, ${ }^{2}$ V. A. Solov'ev, ${ }^{2}$ I. V. Sedova, ${ }^{2}$ \\ G. V. Klimko, ${ }^{2}$ T. A. Komissarova, ${ }^{2}$ S. V. Ivanov, ${ }^{2}$ and S. D. Ganichev ${ }^{1}$ \\ ${ }^{1}$ Terahertz Center, University of Regensburg, Regensburg 93040, Germany \\ ${ }^{2}$ Ioffe Physical-Technical Institute, St. Petersburg 194021, Russia
}

(Received 5 April 2011; accepted 26 July 2011; published online 17 August 2011)

\begin{abstract}
Diluted magnetic semiconductor heterovalent AlSb/InAs/ZnMnTe quantum well (QW) structures with an electron channel have been designed and grown applying molecular-beam epitaxy. The enhanced magnetic properties of QWs as a result of the exchange interaction with $\mathrm{Mn}^{2+}$ ions, are proved by measuring the microwave radiation induced spin polarized electric currents. (C) 2011 American Institute of Physics. [doi:10.1063/1.3624921]
\end{abstract}

The concept of spin-based electronics demands heterostructures possessing high electron mobility, pronounced ferromagnetic properties, and strong spin-orbit interaction (SOI). ${ }^{1,2}$ In particular, manganese doped diluted magnetic semiconductors (DMS) showing high Curie temperature and large Landé factor are in the focus of current research. While enhanced magnetic properties have been obtained in $(\mathrm{Cd}, \mathrm{Mn}) \mathrm{Te}-$ and $(\mathrm{Ga}, \mathrm{Mn})$ As-based quantum wells $(\mathrm{QWs})$, the SOI in these materials is rather small. Thus, realization of DMS heterostructures based on materials which possess a strong SOI, e.g., InAs, becomes important. Most recently, it has been demonstrated that the incorporation of $\mathrm{Mn}$ into a heterostructure device containing an InAlAs/InGaAs QW leads to a two-dimensional hole gas. ${ }^{3}$ In these structures, the $\mathrm{Mn}$ ions are in close proximity to the InGaAs channel hosting the hole gas. While DMS hole systems with strong SOI have been realized and demonstrate very interesting magnetotransport properties, ${ }^{4}$ the fabrication of InAs-based DMS with high mobility two-dimensional electron gas (2DEG) channels is still a challenge. The 2DEG is characterized by a simple parabolic band structure and much higher mobility compared to that of the holes, even in Mn-doped DMS structures like (Cd,Mn)Te QW (Ref. 5) features making 2DEG systems attractive for various applications. The only In(Mn)As-based superlattice with electron mobility $\mu$ from $10^{2}$ to $10^{3} \mathrm{~cm}^{2} / \mathrm{Vs}$ has been realized in Ref. 6 .

Here, we report on the fabrication of Mn modulation doped structures with an InAs 2DEG channel. The QWs were grown applying III-V/II-VI "hybrid" technique following the recipes given in Ref. 7. The Mn layers have been inserted into the II-VI barrier. To explore the magnetic properties of the 2DEG, we investigated spin polarized electric currents induced by microwave (mw) radiation. ${ }^{8,9}$ Our measurements show that hybrid $\mathrm{AlSb} / \mathrm{InAs} /(\mathrm{Zn}, \mathrm{Mn}) \mathrm{Te} \mathrm{QW}$ s are characterized by enhanced magnetic properties which can be changed by tuning of the spatial position of Mn-doping layer as well as by the variation of temperature.

The structures were grown on (001)-oriented GaAs semiinsulating substrates at temperature of $280^{\circ} \mathrm{C}$. For the fabrication of $\mathrm{AlSb} / \mathrm{InAs} /(\mathrm{Zn}, \mathrm{Mn}) \mathrm{Te}$ heterovalent structures with Mn-containing barriers, we used two separated MBE setups. The first, Riber 32P, was employed to obtain the III-V part consisting of the $0.2 \mu$ m-thick GaAs and $2 \mu$ m-thick GaSb buffer layers capped with a 4 nm-thick AlSb barrier and a 15 nm-thick InAs QW (two last layers have common InSb-like interface). A (2.5 nm-GaSb/2.5 nm-AlSb) ${ }_{10}$ superlattice was placed within the first third of the GaSb buffer to suppress propagation of misfit-induced threading dislocations. The II-VI parts of the structures were deposited pseudomorphically on the III-V part in the second two-chambers MBE setup (Semiteq) after the ex-situ sulfur chemical passivation in a $1 \mathrm{M}$ $\mathrm{Na}_{2} \mathrm{~S} 9 \mathrm{H}_{2} \mathrm{O}$ solution of the top InAs layer. The coherent growth of $\mathrm{ZnTe}$ on InAs was initiated by simultaneous opening of $\mathrm{Zn}$ and Te fluxes onto a $(2 \times 4)$ As-reconstructured InAs surface annealed preliminary under an $\mathrm{As}_{4}$ flux in the IIIArsenide chamber of the Semiteq's setup and transferred to the II-VI chamber through ultrahigh vacuum. Such technology results in a high quality $\mathrm{AlSb} / \mathrm{InAs} / \mathrm{ZnTe} \mathrm{QW}$ demonstrating the existence of a 2DEG and quantum confined photoluminescence. ${ }^{11}$ According to X-ray photoelectron spectroscopy of the InAs/ZnTe heterovalent interface grown under similar conditions, its conduction band offset $(\sim 1.65 \mathrm{eV}$, see Ref. 12) is close to that for InAs/AlSb interface.

To demonstrate that the incorporation of Mn leads to enhanced magnetic properties of InAs 2DEG, we prepared series of samples of similar design, shown in Fig. 1(a), but having substantially different density and distribution of $\mathrm{Mn}$ ions in II-VI barriers. Sample A has a 1 ML thick MnTe insertion $(\approx 0.3 \mathrm{~nm})$ separated from the InAs QW by $10 \mathrm{ML}$ of non-magnetic ZnTe, see Fig. 1(a). Sample B has the same spacer and a $10 \mathrm{~nm}$ layer of $\mathrm{Zn}_{0.9} \mathrm{Mn}_{0.1} \mathrm{Te}$ with substantially smaller concentration of Mn per ML. The reference sample $\mathrm{C}$ has no Mn ions.

The 2DEG has the density $n=(1 \div-2) \times 10^{13} \mathrm{~cm}^{-2}$ and the mobility $\mu \sim 5 \times 10^{3} \mathrm{~cm}^{2} / \mathrm{Vs}$ at $T=4.2 \mathrm{~K}$. The electron density is 5 times higher than that in similar InAs-based QWs with AlSb barriers. It indicates that the most of $2 \mathrm{D}$ electrons in a hybrid QW originate from donor centers located at the III-V/II-VI heterovalent interface. Obviously, the surface density of positively charged donor centers should be of order of 2DEG density. It results in a strong asymmetry of QWs in hybrid structures due to a built-in electric field, see Fig. 1. To estimate the energy of size quantization $E_{1}$ and the wave function $\Psi(z)$, we solved the 

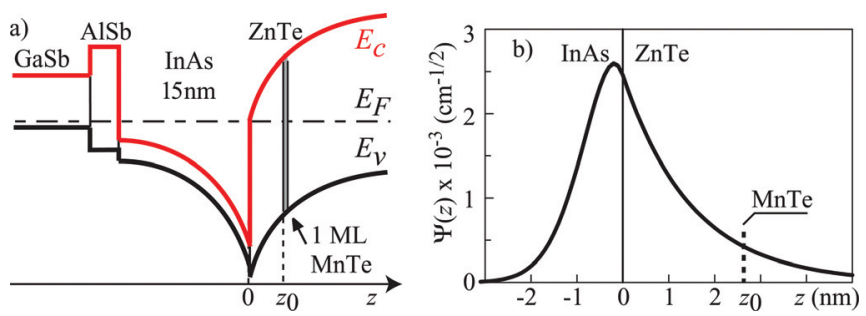

FIG. 1. (Color) (a) Band structure of sample A. (b) Electron wave function $\Psi(z)$ calculated for a triangular QW with the QW potential gradient $1.8 \times 10^{7} \mathrm{eV} / \mathrm{cm}$ resulted from ionized donors at interface with the density $2 \times 10^{13} \mathrm{~cm}^{-2}$, flat barriers, and the effective mass $m^{*}=0.1 m_{0}$. The latter corresponds to $m^{*}$ at conduction-band bottom in ZnTe as well as in InAs with non-parabolicity being taken into account.

Schrödinger equation for an electron in a triangular QW with flat barriers. We found that $E_{1} \sim 1.4 \mathrm{eV}$, being close to $\mathrm{ZnSe}$ conduction-band edge.

The setup used for the measurements of the mw-induced current is shown in Fig. 2. To heat the 2DEG, we applied mw radiation of a Carcinotron operating at frequency $f=290$ $\mathrm{GHz}$ or a Gunn diode $(f=60 \mathrm{GHz})$. The incident power $P \approx 2 \mathrm{~mW}$ was modulated at $330 \mathrm{~Hz}$ by a chopper. Samples of $5 \times 5 \mathrm{~mm}^{2}$ with a pair of Ohmic contacts centered along opposite edges were irradiated by mw radiation at normal incidence. The resulted photocurrent was measured via the voltage drop load resistor applying lock-in technique.

Figure 2 shows the magnetic field dependence of the photocurrent $J_{x}$ induced in samples $\mathrm{A}$ and $\mathrm{C}$. The current increases with $B_{y}$ and reverses its sign as the direction of $B_{y}$ changes (see inset in Fig. 2). The temperature dependence of $J_{x}$ for all samples is plotted in Fig. 3. Both figures indicate a remarkable difference in the photocurrents generated in Mn-doped samples A and B and the reference sample C. At temperatures above $\sim 60 \mathrm{~K}$, the current in all three structures has the same sign and nearly the same magnitude. The analogy, however, disappears as the temperature decreases. In the reference sample $\mathrm{C}$, the polarity of the signal and its

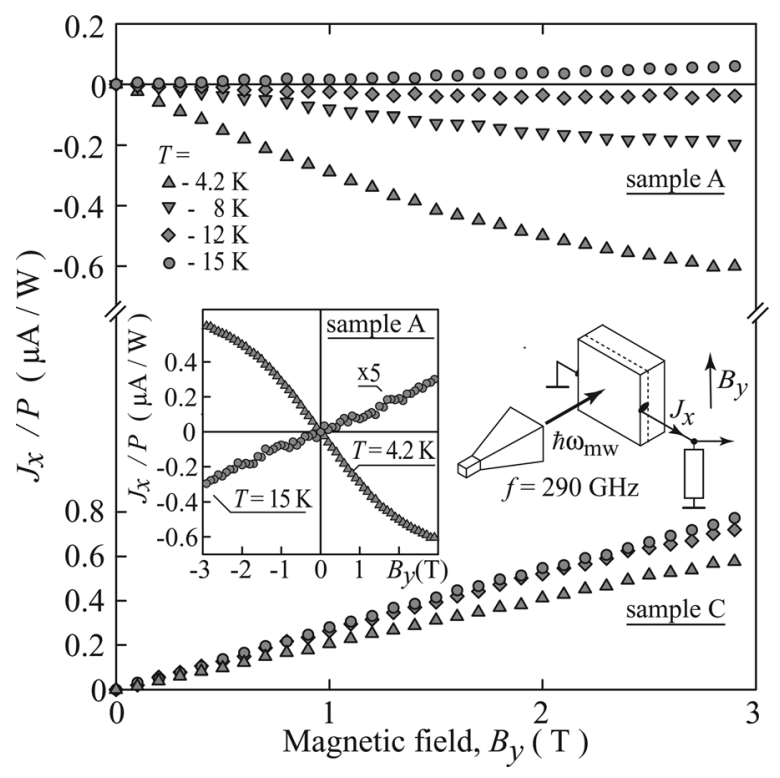

FIG. 2. Magnetic field dependence of $J_{x}(B) / P$ measured for positive $B_{y}$. The inset shows $J_{x}(B) / P$ for both $B_{y}$ directions.

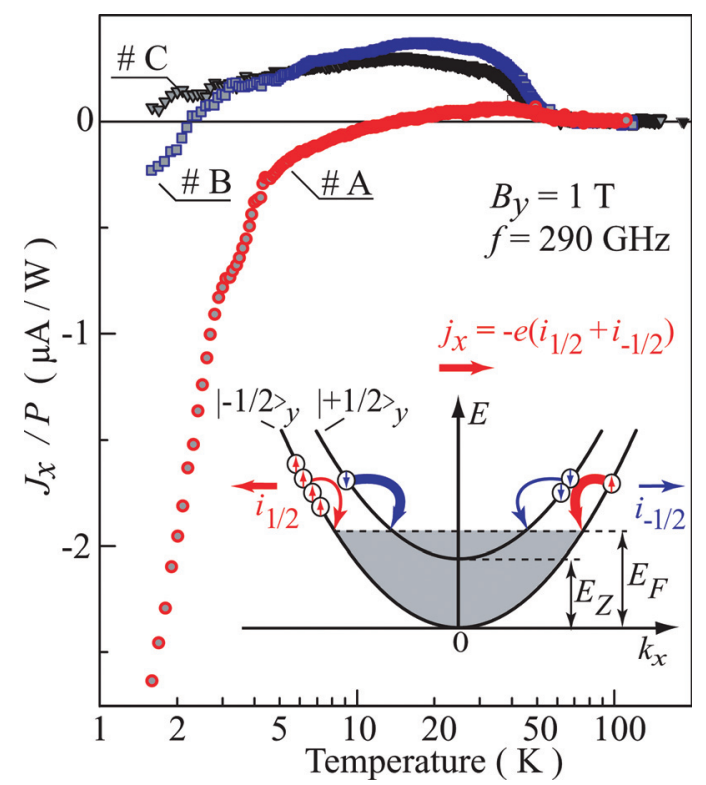

FIG. 3. (Color) Temperature dependence of the photocurrent measured at $B_{y}=1 \mathrm{~T}$. The inset shows the model of the mw-radiation induced spin polarized electric currents. Scattering matrix elements linear in $k$ and $\sigma$ cause asymmetric scattering probabilities sketched by bent arrows of various thickness.

magnetic field behavior remains unchanged. By contrast, in structures doped with $\mathrm{Mn}$, a reduction of temperature results in a sign inversion of the current at $T=T_{\mathrm{inv}}$. In sample A below $T_{\text {inv }} \sim 15 \mathrm{~K}$, the current becomes by more than one order of magnitude larger compared to that measured in samples $\mathrm{B}$ and $\mathrm{C}$ at the same temperature. We also observed that in this sample for $T<8 \mathrm{~K}$, the photocurrent does not depend linearly on $B_{y}$ anymore, and saturates at high magnetic fields (see Fig. 2). In sample B, the inversion temperature is substantially lower $\left(T_{\mathrm{inv}} \sim 2.5 \mathrm{~K}\right)$ and the signal is much smaller than that in sample A.

All these findings give a strong evidence for enhanced magnetic properties of the Mn-doped structures and are well described by the model of the spin-dependent asymmetric energy relaxation of a nonequilibrium 2DEG sketched in the inset in Fig. $3 .^{8-10}$ Excitation of the 2DEG by mw radiation causes electron gas heating. In InAs QWs, the spin-dependent electron-phonon interaction in the energy relaxation results in equal and oppositely directed electron fluxes, $i_{ \pm 1 / 2}$, for opposite spin subbands, $\mid \pm 1 / 2>_{y}$. The application of the magnetic field $B_{y}$ emerges a Zeeman splitting with energy $E_{\mathrm{Z}}$. Thus, the electron densities in the subbands become different, and the fluxes do not compensate each other yielding a net electric current $J_{x} \propto E_{\mathrm{Z}}$.

The coupling of the photocurrent's sign and magnitude to the band spin splitting results in a different behavior of $J_{x}$ in sample $\mathrm{C}$ compared to the Mn-doped samples A and B. In the non-magnetic structure, $E_{\mathrm{Z}}=g \mu_{\mathrm{B}} B$ with $g$ and $\mu_{\mathrm{B}}$ being the $g$ factor and the Bohr magneton, respectively. Consequently, the current increases linearly with rising magnetic field. For Mn-doped samples, a strong temperature dependence of the band spin splitting as well as reversing its sign upon temperature variation is expected for $2 \mathrm{DEG}$ coupled to $\mathrm{Mn}^{2+}$ ions. To estimate the Zeeman splitting in sample A with $\mathrm{Mn}$ ions layer placed at $z=z_{0}$, we use standard expression ${ }^{5}$ 


$$
E_{\mathrm{Z}}=g \mu_{\mathrm{B}} B+\alpha N_{\mathrm{Mn}}\left|\Psi\left(z_{0}\right)\right|^{2} S_{0} \mathrm{~B}_{\frac{5}{2}}\left(\frac{5 \mu_{\mathrm{B}} g_{\mathrm{Mn}} B}{2 k_{\mathrm{B}}\left(T_{\mathrm{Mn}}+T_{0}\right)}\right) .
$$

Here, $g_{\mathrm{Mn}}=2$ is $\mathrm{Mn} g$-factor, $k_{\mathrm{B}}$ the Boltzmann constant, $T_{\mathrm{Mn}}$ the Mn-spin system temperature, parameters $S_{0}$ and $T_{0}$ account for the $\mathrm{Mn}-\mathrm{Mn}$ antiferromagnetic interaction, $\mathrm{B}_{\frac{5}{2}}(x)$ is the modified Brillouin function, $\alpha$ the exchange parameter, and $N_{\mathrm{Mn}}$ the sheet Mn density.

Equation (1) explains well the experimental data for our Mn-doped structures. The effect of the electron exchange interaction with the $\mathrm{Mn}^{2+}$ ions is given by the second term and is most pronounced at low temperatures yielding the giant Zeeman spin splitting. At low $T$, the current following the $E_{\mathrm{Z}}$ is drastically enhanced and saturates at high magnetic fields (see Fig. 2). With the temperature increase, the role of the exchange interaction decreases, $\mathrm{B}_{\frac{5}{2}}(x)$ diminishes, and, for a certain temperature, the intrinsic band spin splitting becomes dominant. Due to the opposite signs of $g$ and $\alpha N_{\mathrm{Mn}}$, the sign of $E_{\mathrm{Z}}$ inverses resulting in the reversion of the photocurrent direction (see Fig. 3). ${ }^{13}$ Lower $T_{\text {inv }}$ and substantially smaller magnitude of the current at $T<T_{\text {inv }}$ detected in sample B in comparison to that of sample A indicate the weaker influence of $\mathrm{Mn}$ on the magnetic properties of the InAs 2DEG channel in this sample.

Our data demonstrate that $\mathrm{Mn}^{2+}$ ions crucially affect the magnetic properties of the InAs 2DEG channel. In both magnetic samples, the Mn doping is done after the InAs QW growth (Fig. 1) and is separated from the QW by rather thick spacer of ZnTe (10 ML), so that the InAs channel is expected to be free of manganese. ${ }^{3,14}$ The latter is also in agreement with the transport data, because diffusion of Mn into the InAs channel should yield a hole gas rather then $2 \mathrm{DEG}$. Thus, we attribute the effect of magnetic ions to the exchange interaction caused by the penetration of electron wave function into the barrier. ${ }^{15}$ Since the energy level $E_{1}$ is close the conduction band bottom of II-VI barrier, the electron envelope wave function deeply penetrates into ZnTe resulting in an efficient exchange interaction, see Fig. 1(b). Estimations for sample A show that at $T=1.8 \mathrm{~K}$ and $B=2 \mathrm{~T}$ exchange spin splitting is one order of magnitude larger than the intrinsic Zeeman splitting. ${ }^{16,17}$ In sample $\mathrm{B}$, the overlap of the electron function with the $\mathrm{Mn}^{2+}$ ions is substantially smaller because of spatial distribution of the $\mathrm{Mn}^{2+}$ ions over larger distance. Both, the strong enhance of spin splitting in sample A and relatively weak exchange interaction in sample B are in agreement with photocurrent measurements, see Fig. 3.

Summarizing, we show that heterostructures characterized by a giant Zeeman splitting in $n$-type InAs QW and showing behavior typical for DMS can be obtained by growing of III-V/II-Mn-VI coherent "hybrid" heterostructures with the Mn insertion to the II-VI barrier. Our measurements demonstrate that the enhanced magnetic properties are due to the penetration of electronic wave function into the ( $\mathrm{Zn}, \mathrm{Mn}) \mathrm{Te}$ layer and can be controllably varied by the position and density of $\mathrm{Mn}^{2+}$ ions.

The financial support from the DFG (SFB 689 and Priority Group 1483), the Linkage Grant of IB of BMBF at DLR, RFBR, and Russian Ministry of Education and Sciences is gratefully acknowledged. We are grateful to D. R. Yakovlev and B. Aronzon for fruitful discussions.

${ }^{1}$ Spintronics in Semiconductors and Semimetals series, edited by, T. Dietl, D. Awschalom, and M. Kaminska (Academic, London, 2008).

${ }^{2}$ Spin Physics in Semiconductors, edited by M. I. Dyakonov (Springer, Berlin, 2009).

${ }^{3}$ U. Wurstbauer, M. Soda, R. Jakiela, D. Schuh, D. Weiss, J. Zweck, and W. Wegscheider, J. Cryst. Growth 311, 2160 (2009).

${ }^{4}$ U. Wurstbauer, C. Sliwa, D. Weiss, T. Dietl, and W. Wegscheider, Nature Phys. 6, 955 (2010).

${ }^{5}$ J. K. Furdyna, J. Appl. Phys. 64, R29 (1988).

${ }^{6}$ G. A. Khodaparast, M. A. Zudov, J. Kono, Y. H. Matsuda, T. Ikaida, S. Ikeda, N. Miura, T. Slupinski, A. Oiwa, H. Munekata, G. D. Sanders, Y. Sun, and C. J. Stanton, J. Supercond 16, 107 (2003).

${ }^{7}$ S. V. Ivanov, V. A. Kaygorodov, S. V. Sorokin, V. A. Solov'ev, A. A. Sitnikova, O. G. Lyublinskaya, Ya. V. Terent'ev, Yu. B. Vasilyev, V. L. Berkovits, A. A. Toropov, and P. S. Kop'ev, Phys. status solidi C 1, 1468 (2004).

${ }^{8}$ S. D. Ganichev, S. A. Tarasenko, V. V. Bel'kov, P. Olbrich, W. Eder, D. R. Yakovlev, V. Kolkovsky, W. Zaleszczyk, G. Karczewski, T. Wojtowicz, and D. Weiss, Phys. Rev. Lett. 102, 156602 (2009).

${ }^{9}$ C. Drexler, V. V. Bel'kov, B. Ashkinadze, P. Olbrich, C. Zoth, V. Lechner, Ya. V. Terent'ev, D. R. Yakovlev, G. Karczewski, T. Wojtowicz, D. Schuh, W. Wegscheider, and S. D. Ganichev, Appl. Phys. Lett. 97, 182107 (2010).

${ }^{10}$ S. D. Ganichev, S. N. Danilov, V. V. Bel'kov, S. Giglberger, S. A. Tarasenko, E. L. Ivchenko, D. Weiss, W. Jantsch, F. Schäffler, D. Gruber, and W. Prettl, Phys. Rev. B 75, 155317 (2007).

${ }^{11}$ S. V. Ivanov, O. G. Lyublinskaya, Yu. B. Vasilyev, V. A. Kaygorodov, S. V. Sorokin, I. V. Sedova, V. A. Solov'ev, B. Ya. Meltser, A. A. Sitnikova, T. V. L'vova, V. L. Berkovits, A. A. Toropov, and P. S. Kop'ev, Appl. Phys. Lett. 84, 4777 (2004).

${ }^{12}$ Th. Gleim, L. Weinhardt, Th. Schmidt, R. Fink, C. Heske, E. Umbach, P. Grabs, G. Schmidt, L. W. Molenkamp, B. Richter, A. Fleszar, and H. P. Steinrück, Appl. Phys. Lett. 81, 3813 (2002).

${ }^{13}$ We note, that a similar $J_{x}(T)$ behavior has also been observed recently in $(\mathrm{Cd}, \mathrm{Mn}) \mathrm{Te} /(\mathrm{Cd}, \mathrm{Mg}) \mathrm{Te} \mathrm{QWs}$ where, in contrast to the structures studied here, $(\mathrm{Cd}, \mathrm{Mn}) \mathrm{Te}$ thin layers were directly implemented in the QW.

${ }^{14}$ G. Prechtl, W. Heiss, A. Bonanni, W. Jantsch, S. Mackowski, and E. Janik, Phys. Rev. B 68, 165313 (2003).

${ }^{15}$ E. Z. Meilikhov and R. M. Farzetdinova, JETP 110, 794 (2010).

${ }^{16}$ The estimation is made after Eq. (1) for $N_{M n}=10^{15} \mathrm{~cm}^{-2}$ and $\alpha=10^{-20}$

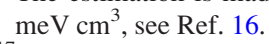

${ }^{17}$ A. Twardovski, P. Swiderski, M. V. Ortenberg, and R. Pauthenet, Solid State Commun. 50, 509 (1984). 